\title{
Effects of doping on the kinetics of laser-induced low-temperature crystallization of amorphous silicon
}

\author{
Yu. L. Khait, R. Beserman, ${ }^{\text {a) }}$ and A. Chack \\ Solid State Institute and Department of Physics, Technion-Israel Institute of Technology, Haifa 32000, Israel \\ W. Beyer \\ Institute für Photo-Voltaik Forschungzentrum, Jülich 52425, Germany
}

(Received 29 June 2004; accepted 29 March 2005; published online 20 June 2005)

\begin{abstract}
Substantial effects of boron and phosphorus doping on the kinetics of laser-induced crystallization (LIC) in hydrogenated amorphous silicon $(a-\mathrm{Si}: \mathrm{H})$ are reported. A kinetic nanoscopic electron-related LIC model that suggests predictions and explanations of observed effects of B and $\mathrm{P}$ doping on the LIC temperatures and crystallite size in $a-\mathrm{Si}: \mathrm{H}$ is presented. The LIC is considered to be the integral effect of a huge number of nanoscale picosecond material reconstructions, each of which is generated by a nanoscopic short-lived (picosecond) large-energy fluctuation. The LIC in doped $a$-Si:H occurs at temperatures substantially lower than those found in the crystallization in a furnace. Crystallite size in B-doped $a-\mathrm{Si}: \mathrm{H}$ is half of that in P-doped and undoped material. (C) 2005 American Institute of Physics. [DOI: 10.1063/1.1921337]
\end{abstract}

\section{INTRODUCTION}

In the past, laser-induced phase transition from amorphous to crystalline phase in silicon has been extensively studied by various experimental techniques. ${ }^{1-4}$ The in situ Raman data from laser-heated silicon were reported long ago $^{5}{ }^{5}$ in situ ellipsometry monitored the nucleation of microcrystalline silicon $\left(\mu c\right.$-Si). ${ }^{6}$ More recently, it has been found that under laser illumination, a high concentration of excited electrons weakens the covalent bond, resulting in a melting transition to a metallic state. ${ }^{7}$

The effect of impurities on the crystallization of amorphous silicon has been given a lot of attention due to its industrial applications. Noble gases as well as $\mathrm{O}$ and $\mathrm{N}$ have been found to retard the growth process. ${ }^{8}$ Dopant impurities such as B, P, or As, on the other hand, favor the regrowth of amorphous silicon $(a-\mathrm{Si}){ }^{9-12}$ The diffusion and the role of impurities in the annealing process have been studied in great detail. A nonequilibrium theory based on short-lived fluctuations has been proposed, ${ }^{13}$ but, on the other hand, stress and electric field are the main factors in the impurityinduced regrowth. ${ }^{9}$ Under equilibrium conditions, vacancies and interstitials mediate the diffusion mechanism of dopants in $\mathrm{Si}^{14}{ }^{14} \mathrm{~A}$ nonequilibrium mechanism explains the enhanced crystallization when amorphous silicon is illuminated by a $600-\mathrm{keV} \mathrm{Kr}^{++}$irradiation. ${ }^{15}$ A review of the kinetics of solidphase crystallization in $a$-Si was presented by Olson and Roth, ${ }^{3}$ who focused their work on solid-phase epitaxy.

In spite of all the studies, the mechanism and the kinetics of the nonequilibrium phenomena involved in laser-induced crystallization (LIC) of amorphous materials are not yet fully understood.

Demonstrations of solar cell operation for cells prepared from amorphous or microcrystalline hydrogenated silicon attracted a lot of attention. ${ }^{14}$ At the annealing temperatures

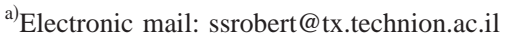

considered here, most of the hydrogen is gone and, for all practical purposes, we are dealing with hydrogen-free $a$-Si. ${ }^{16}$

One of the main difficulties is associated with the highly nonequilibrium character of the LIC-related phenomena, where traditional methods of thermodynamics and the equilibrium theory are not effective. Some aspects of this problem were discussed in our article on the $\mathrm{LIC}^{17}$ and on the structural changes induced by $\mathrm{cw}$ laser ${ }^{18}$ in undoped $a-\mathrm{Si}: \mathrm{H}$ (and in other materials). This consideration shows that the LIC includes the following nonequilibrium athermal and thermal phenomena: First, the formation by laser beams of high nonequilibrium electron and hole concentrations, $n_{e}$ and $n_{h}$; second, the effect of these high electron and hole concentrations on the nonequilibrium material reconstructions involved in the LIC; third, effects of dopants on the LIC kinetics; fourth, the influence of the effective kinetic temperature of the material irradiated by laser beams. We have suggested a nanoscopic kinetic electron-related model for the consideration of the nonequilibrium phenomena involved in the LIC and the structural changes in undoped amorphous materials at relatively low temperatures. ${ }^{17-19}$ This model, extended here to the description of the LIC in doped $a-\mathrm{Si}: \mathrm{H}$, proposes predictions, some of which have been confirmed experimentally. In this letter we present a brief report on recent experimental and theoretical studies on the effects of boron and phosphorus doping on the LIC in $a-\mathrm{Si}: \mathrm{H}$. Raman spectroscopy is employed for the experimental study of the LIC in B-and P-doped $a-\mathrm{Si}: \mathrm{H}$.

\section{DISCUSSION}

We shall utilize here some earlier results on the nanoscopic kinetic model applied to various processes and materials. ${ }^{18-24}$ These results suggest the following generic nanoscopic kinetic approach to the nonequilibrium LICrelated phenomena in doped and undoped materials: the observed material changes appear to be the integral effect of a 
huge number of nanoscale "elementary" nonequilibrium picosecond events (NENEPEs), each of which involves atomic and electronic interrelated reconstructions in the metastable amorphous material, driving it to a more stable state related to a lower free energy. Every NENEPE and the related local picosecond material reconstructions are generated by a nanoscopic short-lived (picosecond) large-energy fluctuation (SLEF) of a small number $N_{0} \geqslant 1$ of strongly fluctuating hyperthermal atoms of thermal energy $\epsilon_{\mathrm{up}} \geqslant \Delta E \gg k T$. ${ }^{17-24} \mathrm{~A}$ single NENEPE includes diffusionlike hoppings of strongly fluctuating $\mathrm{Si}$ atoms over energy barriers $\Delta E \gg k T$ into new positions during the SLEF lifetime $\Delta \tau=10^{-13}-10^{-12} \mathrm{~s}$. The hoppings are accompanied by the cutting of some interatomic prehopping bonds of the hopping atoms with its neighbors in the prehopping positions and establishing new (electron-mediated) bonds with new neighbors after the hoppings. It is assumed that the LIC involves $\nu_{A}=5-10$ (per atom) SLEF-induced hoppings of Si atoms. ${ }^{17-19}$

Individual SLEFs create strong local nanoscale lattice distortions that form transient point dynamic defects of picosecond duration and nanometer size. ${ }^{19-24}$ These defects generate the strong electron-lattice interaction that produces $\delta n^{d}$ nonadiabatic downward electron transitions accompanying atomic hoppings. ${ }^{17-23}$ The $\delta n^{d}$ downward electron transitions reduce the hopping activation energy $\Delta E=E-\delta n_{e}^{d}\langle\Delta e\rangle$, and thus enhance exponentially the hopping probability, which has been calculated from the kinetic consideration of related picosecond SLEF-induced nanoscale nonequilibrium phenomena. Here $E$ is the energy barrier, which should be overcome without the "assistance" of the downward electron transitions (i.e., when $\delta n^{d} \rightarrow 0$ ); $\langle\Delta e\rangle$ is the energy reduction per transition. This reduces exponentially the nucleation time

$$
t_{N}=\nu_{A} \Delta \tau \exp \left(\Delta G / k T_{A}\right) .
$$

Here $\Delta G=\Delta E-T_{A} \Delta S=E-\delta n e\langle\Delta e\rangle-T_{A} \Delta S$ and $\Delta S$ are the related local changes in the effective activation free energy, and local entropy $T_{A}$ is the effective local temperature in the irradiated material region, which can be measured by the Raman Stokes-anti-Stokes ratios. This model leads to some verifiable predictions on the effect of doping on the LIC in $a-\mathrm{Si}: \mathrm{H}$, confirmed by measurements (Figs. 1 and 2 and Table I). Equation (1) shows that the crystallization time $t_{N}$ depends exponentially on the energy $E$, the number $\delta n_{e}^{d}$ of downward electron transitions, and the temperature $T_{A}$ in the laser spot-related volume $V_{L} \approx \pi R_{L}^{2} \ell$ of radius $R_{L}$ and thickness $\ell$ determined by the photon penetration depth.

The $1 \%$ boron impurity concentration should increase $T_{A}$ substantially, due to the following factors: In undoped and doped $a-\mathrm{Si}: \mathrm{H}$ the temperature $T_{A}$ is determined by (a) the initial material temperature $T$, (b) the laser input energy density per unit of time, and (c) the heat conductivity that determines the energy fluxes from the volume $V_{L}$ into the "cold" surroundings. The heat conductivity in the undoped material, $æ$, and the B-doped samples, $\alpha_{\mathrm{B}}$ are determined mainly by those of electron-hole plasma, which are greater than the phonon conductivity. Here $æ$ and $a_{\mathrm{B}}$ depend on related ambipolar diffusivities, $\delta$ and $\delta_{\mathrm{B}}$, of the electron-hole plasmas, respectively. In undoped $a-\mathrm{Si}: \mathrm{H}$ laser-generated mobile electrons and holes of concentrations $n_{e}$ and $n_{h}$ have a rather high

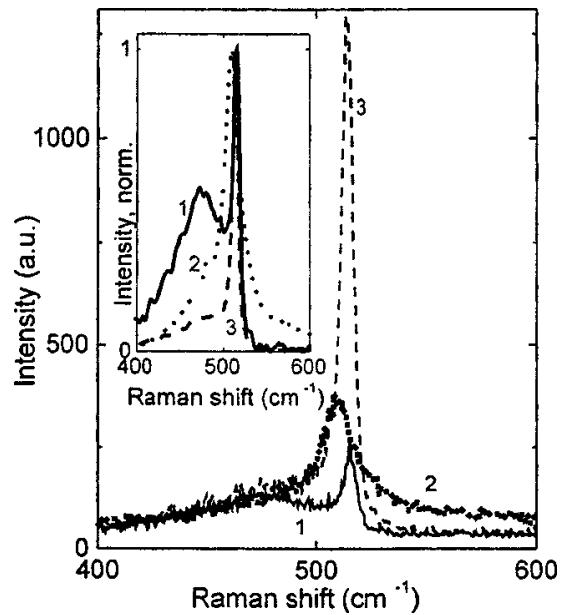

FIG. 1. Raman spectra of (1) undoped $a$-Si:H, (2) $1 \%$ B-doped $a-\mathrm{Si}: \mathrm{H}$, and (3) $1 \%$ P-doped $a-\mathrm{Si}: \mathrm{H}$ after the crystallization induced by a laser at a power of $10 \mathrm{~mW}$ for 10 mins. Inset presents the Raman spectra normalized by their maxima.

$\delta$ and $æ$ that lead to the temperature $T_{A} \approx 600 \mathrm{~K}$ in $V_{L}{ }^{17}$ Doping by $1 \% \mathrm{~B}$ produces high concentrations, $n_{e \mathrm{~B}} \gg n_{e}$ and $n_{h \mathrm{~B}} \gg n_{h} \quad$ (e.g., $n_{e} \approx n_{h} \approx 10^{18} \mathrm{~cm}^{3} \quad$ and $n_{e \mathrm{~B}} \approx n_{h \mathrm{~B}} \approx 10^{20}$ $-10^{21} \mathrm{~cm}^{-3}$ ), of immobile electrons trapped by $\mathrm{B}$ dopants and holes interacting strongly with the trapped electrons. Such interaction reduces considerably the hole mobility, ambipolar diffusivity $\delta_{\mathrm{B}}$, and the related heat conductivity $a_{\mathrm{B}}$ $<a$ of the electron-hole plasma in B-doped $a-\mathrm{Si}: \mathrm{H}$. This causes a marked enhancement in the local temperature $T_{A \mathrm{~B}}$ $>T_{A}$ in the B-doped $a-\mathrm{Si}: \mathrm{H}$. Temperature measurements confirm the above conclusion: $T_{A \mathrm{~B}} \approx 770 \mathrm{~K}$, whereas $T_{A}$ $\approx 600 \mathrm{~K}$. (Table I) Higher $T_{A \mathrm{~B}}$ leads to the exponential reduction of the crystallization time $t_{N}$ [Eq. (1)] as compared to that in the undoped material.

The next effect of boron doping is a reduction of energy $E$ in Eq. (1) by the creation of a high concentration of holes and trapped excited valence electrons that weakens $\mathrm{Si}-\mathrm{Si}$ interatomic bonds. This reduces $\Delta E$ and $\Delta G$ in Eq. (1), and

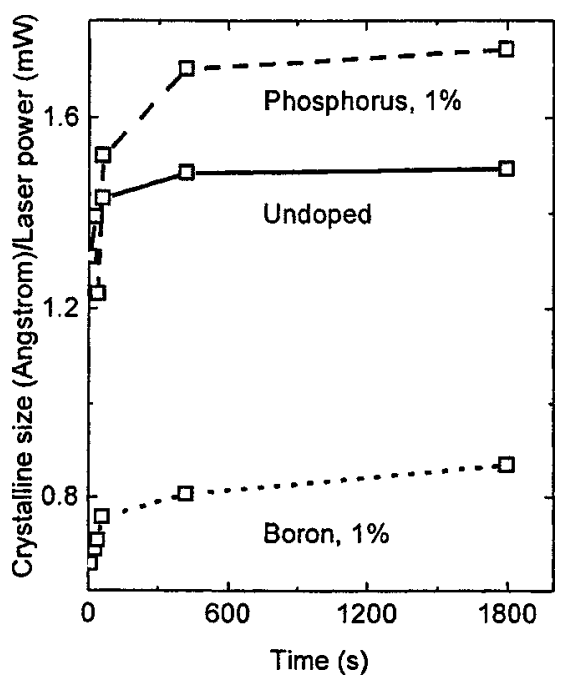

FIG. 2. Crystallite sizes (normalized by the laser power) as a function of time, obtained in the course of laser-induced crystallization in undoped, $1 \%$ B-doped and 1\% P-doped $a-\mathrm{Si}: \mathrm{H}$. 
TABLE I. Comparison between standard oven annealing and laser annealing for undoped $a$-Si:H and phosphorus- and boron-doped $a$-Si:H. The average size of the microcrystals is deduced from the Raman shift of the crystalline Si line.

\begin{tabular}{|c|c|c|c|}
\hline & $\begin{array}{l}a-\mathrm{Si}: \mathrm{H} \\
\text { undoped }\end{array}$ & $\begin{array}{c}a-\mathrm{Si}: \mathrm{H} \\
1 \% \mathrm{P}\end{array}$ & $\begin{array}{c}a-\mathrm{Si}: \mathrm{H}, \\
1 \% \mathrm{~B}\end{array}$ \\
\hline $\begin{array}{l}\text { Oven temperature of } \\
\text { crystallization }(\mathrm{K})\end{array}$ & 940 & 930 & 880 \\
\hline $\begin{array}{l}\text { Laser temperature of } \\
\text { crystallization }(\mathrm{K})\end{array}$ & 610 & 590 & 770 \\
\hline $\begin{array}{l}\text { Microcrystalline size, oven } \\
\text { crystallization }(\AA)\end{array}$ & 29 & 24 & 15 \\
\hline $\begin{array}{l}\text { Microcrystalline size, } \\
\text { laser }(\AA)\end{array}$ & 33 & 27 & 15 \\
\hline
\end{tabular}

thus shortens exponentially the nucleation time $t_{N}$. Besides, the reduction of $E$ caused by B doping softens slightly the material, and thus reduces a bit the Raman peak frequency. This should cause a small shift of the Raman peak towards lower frequency that is confirmed by measurements (Fig. 1 and Table I).

Thus, in B-doped $a-\mathrm{Si}: \mathrm{H}$ the values of $\Delta G, \Delta E$, and $T_{A}$ in Eq. (1) should be replaced with new parameters $\Delta G_{\mathrm{B}}$, $\Delta E_{\mathrm{B}}$, and $T_{A \mathrm{~B}}$. Here $\Delta G_{\mathrm{B}}=\Delta G-\Delta g_{\mathrm{B}}<\Delta G$, where $\Delta G_{\mathrm{B}}$ $=\Delta E_{\mathrm{B}}-T_{A \mathrm{~B}} \Delta S_{\mathrm{B}}$ is determined by the lower activation energy $\Delta E_{\mathrm{B}}=\Delta E-\Delta U_{\mathrm{B}}<\Delta E$ and higher $T_{A \mathrm{~B}}>T_{A} ; \Delta U_{\mathrm{B}}$ is the reduction in $\Delta E$. The value $\Delta g_{\mathrm{B}}=\Delta G-\Delta G_{\mathrm{B}} . \Delta g_{V}$ can be estimated roughly from the differences $\Delta g_{T \mathrm{~B}}=\Delta G_{T}-\Delta G_{T \mathrm{~B}}$ in the activation free energies and temperatures $T_{\mathrm{B}}-T=-60 \mathrm{~K}$ in the thermal crystallization in undoped and B-doped $a$ -Si:H taking place at temperatures $T=940 \mathrm{~K}$ and $T_{\mathrm{B}}$ $\approx 880 \mathrm{~K}<T$, respectively (Table I). Using the equations for the nucleation time in the thermal crystallization in $a-\mathrm{Si}: \mathrm{H}$ and $a-\mathrm{Si},{ }^{16}$ we find $\Delta g_{T \mathrm{~B}} \approx 0.07 \Delta G_{T}$ and $\Delta G_{T \mathrm{~B}} \approx 0.93 \Delta G_{T}$. Assuming that approximately similar reduction in $\Delta G_{\mathrm{B}}$ $\approx 0.93 \Delta G$ is caused by B doping in the LIC in $a-\mathrm{Si}: \mathrm{H}$, we find $\Delta G_{\mathrm{B}} \approx 1.62 \mathrm{eV}$, where we use $\Delta G \approx 1.75 \mathrm{eV}$ obtained for the LIC in undoped $a-\mathrm{Si}: \mathrm{H}^{17}$ Taking this value of $\Delta G_{\mathrm{B}}$ and $T_{A \mathrm{~B}} \approx 770 \mathrm{~K}$ one estimates [from Eq. (1)] $t_{N_{\mathrm{B}}} \approx 0.1 \mathrm{~s}$.

The discussed effects of $\mathrm{B}$ doping enable us to make the following "controversial" prediction confirmed experimentally (Figs. 1 and 2, Table I) and explained below: boron doping, which enhances strongly the LIC rate coefficient $K_{N_{\mathrm{B}}} \approx t_{N_{\mathrm{B}}}^{-1}$, reduces substantially the size of crystallites compared to that in undoped $a-\mathrm{Si}: \mathrm{H}$ due to the following arguments. A high density of small Si-crystalline nuclei is formed during a short time interval (e.g., during $0.1 \mathrm{~s}$ ) after the beginning of the LIC in B-doped $a-\mathrm{Si}: \mathrm{H}$. The further substantial growth of the initial small Si nuclei is prevented by their random orientation and the unfavorable composition of the intercrystallite material, which contains a relatively low $\mathrm{Si}$ concentration and a high concentration of impurities. As a result, after insubstantial short-term growth the $\mathrm{Si}$ nuclei should cease to grow; similar arguments enable one to expect a substantial reduction of the crystallite size in the thermal crystallization in B-doped $a-\mathrm{Si}: \mathrm{H}$. The size of crystallites was measured by the Raman technique, as proposed in Ref. 23.

\section{EXPERIMENTAL RESULTS}

Experimental data presented in Table I and in Fig. 2 confirm the above conclusions and show that the size of crystallites in B-doped $a-\mathrm{Si}: \mathrm{H}$ is about two times smaller than that in undoped $a-\mathrm{Si}: \mathrm{H}$. This leads to another observable consequence: the B-doped material contains a relatively large amount of disordered intercrystallite substances, and therefore the Raman spectrum of this material should contain large "tails" related to the disordered material phase. The Raman peak related to the crystalline phase is expected to be not very high and narrow. The experimental data presented in Fig. 1 confirm these expectations.

Our model predicts that contrary to the B-doped material, doping of $a-\mathrm{Si}: \mathrm{H}$ by $1 \%$ of phosphorus should not change markedly the local effective temperature $T_{A}$ and energy $E$ in Eq. (1) as well as the size of the crystallite compared to the undoped material. This conclusion, confirmed experimentally (Figs. 1 and 2, Table I), results from the following factors. $1 \% \mathrm{P}$ doping enhances considerably the concentration of mobile electrons, but does not change markedly the hole concentration created by laser irradiation. Therefore, the ambipolar diffusivity $\delta_{\mathrm{P}} \approx \delta$ and heat conductivity $a_{\mathrm{P}}$ $\approx a$ of the electron-hole plasma as well as temperature $T_{A \mathrm{P}}$ $\approx T_{A}$ in the $\mathrm{P}$-doped material are approximately equal to their counterparts in the undoped material (Table I). In P-doped $a-\mathrm{Si}: \mathrm{H}$ the $\mathrm{Si}$ crystallite size is markedly larger than that of B-doped $a$-Si:H and comparable to that of undoped $a$-Si:H (Table I and Fig. 2), since P doping produces considerably lower initial crystalline density compared to that in the B-doped material. As a result the initial crystalline nuclei in P-doped $a-\mathrm{Si}: \mathrm{H}$, which are separated by greater distances, weakly affect one another before they become rather large. In addition, the intercrystallite substances between the separated nuclei contain a greater amount of silicon needed for the nuclei growth compared to that in B-doped $a$-Si:H. At the same time, phosphorus doping does not "soften" the material, and thus does not shift markedly the Raman peak, compared to undoped $a-\mathrm{Si}: \mathrm{H}$. This conclusion is also in agreement with experimental data (Table I and Fig. 1).

The standard model is that in disordered semiconductors, electrical transport occurs between localized states near the Fermi level $\left(E_{F}\right)$. This transport depends on the electronic density of states, on the position of $E_{F}$, and on the temperature. ${ }^{25-27}$ A detailed study of the influence of isoelectronic and Coulombic impurities on the entropy on the impurity levels has been proposed ${ }^{28}$ based on the Fermi level shift.

The models developed until now calculate the impuritylevel energy or the shift of $E_{F}$ as a function of the impurity type and concentration, under equilibrium conditions. In our model we propose a mechanism which explains how any single atom can overcome the energy barrier when jumping from a given position to a new one, which is more favorable energetically.

In the past this model has been used to explain, among other phenomena, the diffusion of impurities in silicon, ${ }^{13}$ the crystallization of amorphous silicon, ${ }^{16}$ low-temperature LIC, ${ }^{17}$ and structural changes in amorphous materials. ${ }^{18}$ 


\section{CONCLUSIONS}

The following results for the LIC in B- and P-doped $a-\mathrm{Si}: \mathrm{H}$ are obtained: (i) The observed LIC is the integral effect of a huge number of nanoscale picosecond thermalathermal atomic and electronic reconstructions towards more ordered material states. (ii) Boron doping enhances markedly the local LIC temperature in a laser-affected volume compared to that in the LIC in undoped $a-\mathrm{Si}: \mathrm{H}$; but this temperature is still substantially lower and the LIC is much faster compared to the furnace crystallizations. (iii) The LIC in P-doped $a-\mathrm{Si}: \mathrm{H}$ is much faster than those in the furnace crystallizations and occurs at temperatures markedly lower than those in the LIC in the B-doped material. (iv) The crystallite size produced by the LIC in B-doped $a-\mathrm{Si}: \mathrm{H}$ is substantially smaller than that in the P-doped and undoped materials. (v) B doping shifts the Raman peak towards lower frequencies, whereas P doping does not shift the Raman peak.

\section{ACKNOWLEDGMENTS}

This research was supported in part by grants from the Bundensministerium für Bildung, Forschung and Technology (BMBF), Germany through the Israel Ministry of Science and the Israel Ministry of Absorption.

${ }^{1}$ Laser-Solid Interactions and Transient Thermal Processing of Materials, edited by J. Narayan, W. L. Brown, and R. A. Lemons (Elsevier, New York, 1983).

${ }^{2}$ Energy Beam-Solid Interactions in Transient Thermal Processing, edited by J. C. C. Fan and N. M. Johnson (Elsevier, New York, 1984).

${ }^{3}$ G. L. Olson and J. A. Roth, Mater. Sci. Rep. 3, 1 (1988).

${ }^{4}$ G. M. Ferreira, A. S. Ferlando, C. Chen, R. J. Koval, J. M. Pearce, C. Ross, C. R. Wronski, and R. W. Collins, J. Non-Cryst. Solids 338-340, 13 (2004).

${ }^{5}$ H. W. Lo and A. Compaan, J. Appl. Phys. 51, 1565 (1980).
${ }^{6}$ B. Drevillon, C. Godet, and S. Kumar, Appl. Phys. Lett. 50, 1651 (1987).

${ }^{7}$ R. L. Silverstrelli, A. Alavi, M. Porrinello, and D. Frenkel, Phys. Rev. Lett. 77, 3149 (1996).

${ }^{8}$ E. F. Kennedy, L. Csepregi, J. W. Mayer, and T. W. Sigmon, J. Appl. Phys. 48, 4241 (1977).

${ }^{9}$ I. Suni, G. Göltz, M. G. Grimaldi, M. A. Nicolet, and S. S. Low, Appl. Phys. Lett. 40, 269 (1982).

${ }^{10}$ A. Lietoila, A. Wakita, T. W. Sigmon, and J. F. Gibbons, J. Appl. Phys. 53, 4399 (1982)

${ }^{11}$ H. Ishiwara, A. Tamba, H. Yamamoto, and S. Furnkawa, Jpn. J. Appl. Phys., Part 2 24, L513 (1985).

${ }^{12}$ R. Saleh and N. H. Nickel, J. Non-Cryst. Solids 338-340, 143 (2004).

${ }^{13}$ Yu. L. Khait, R. Brener, and R. Beserman, Phys. Rev. B 38, 6107 (1988).

${ }^{14}$ C. S. Nicols, C. G. Van de Walle, and S. T. Pantelides, Phys. Rev. B 40, 5484 (1989).

${ }^{15}$ J. S. Custer, A. Battaglia, M. Soggio, and F. Priolo, Phys. Rev. Lett. 69, 780 (1992).

${ }^{16}$ J. Meier, R. Flückiger, H. Keppner, and A. Shah, Appl. Phys. Lett. 65, 860 (1994).

${ }^{17}$ Yu. L. Khait and R. Beserman, Phys. Rev. B 33, 2983 (1986).

${ }^{18}$ Yu. L. Khait, R. Beserman, A. Chack, R. Weil, and W. Beyer, Appl. Phys. Lett. 81, 3347 (2002).

${ }^{19}$ I. Abdulhalim, R. Beserman, Yu. L. Khait, and R. Weil, Appl. Phys. Lett. 51, 898 (1987).

${ }^{20}$ Yu. L. Khait, Kinetics and Application of Atomic Diffusion in Solids: Nanoscopic Electron-Affected Stochastic Dynamics (SCITECH, Switzerland, 1997).

${ }^{21}$ Yu. L. Khait, R. Beserman, W. Beyer, R. Weil, and H. Wagner, Phys. Rev. B 42, 9000 (1990).

${ }^{22}$ Yu. L. Khait, A. Silverman, R. Weil, and J. Adler, Phys. Rev. B 44, 8308 (1991).

${ }^{23}$ K. R. Rao, Curr. Sci. 75, 1328 (1998).

${ }^{24}$ Yu. L. Khait, J. Salzman, and R. Beserman, Appl. Phys. Lett. 53, 2135 (1988).

${ }^{25}$ Ch. Ossadnic, S. Veprek, and I. Gregora, Thin Solid Films 337, 148 (1999).

${ }^{26}$ N. F. Mott and E. A. Davis, Electronic Processes in Non-Crystal Materials, 2nd ed. (Oxford University, Oxford, 1979).

${ }^{27}$ C. Godet, J. Non-Cryst. Solids 299-302, 333 (2002); S. B. Concari, R. H. Bruitago, M. T. Gutierrez, and J. J. Gandia, J. Appl. Phys. 94, 2417 (2003).

${ }^{28}$ J. A. Van Vechten and C. D. Thurmond, Phys. Rev. B 14, 3539 (1976). 